\title{
The 1.3-year and 156-day periodicities in sunspot data: Wavelet analysis suggests a common origin
}

\author{
N. A. Krivova ${ }^{1,2}$ and S. K. Solanki ${ }^{1}$ \\ 1 Max-Planck-Institut für Aeronomie, 37191 Katlenburg-Lindau, Germany \\ e-mail: natalie@linmpi.mpg.de; solanki@linmpi.mpg.de \\ 2 On leave from Astronomical Institute, St. Petersburg University, 198504 St. Petersburg, Russia
}

Received 14 May 2002 / Accepted 11 July 2002

\begin{abstract}
Helioseismic data have revealed a 1.3-year periodicity in the solar rotation rate near the bottom of the solar convection zone. In order to test whether these rotation rate variations have a significant impact on the solar dynamo, we search for such a periodicity in tracers of relatively freshly emerged flux at the solar surface, namely sunspots. Sunspot areas and sunspot number time series are studied with the help of the wavelet transform. Significant power at this period (1.28 years) is indeed found and is observed to vary strongly with time. This provides independent support for the presence of a 1.3 year periodicity in solar data. The power at the 154-158-day Rieger period of solar flares is seen to vary approximately in phase with the 1.28-year period. Based on this we propose that the Rieger period is the third harmonic $(3 \times 156$ days $=1.28$ years $)$ of the 1.3 -year period. If the rotation rate of the Sun does vary with 1.3 years then the enhanced flaring with the Rieger period may finally be driven by the 1.3 year periodicity. However, the power in both periods is also found to approximately follow the total number of sunspots. Therefore we cannot rule out that the 1.3-year and 156-day periods are harmonics of the solar activity cycle. Finally, our analysis of a calibrated sunspot area record reveals that the 156-day period continues into the most recent cycles, in contrast to earlier results.
\end{abstract}

Key words. Sun: interior - Sun: magnetic fields - Sun: sunspots

\section{Introduction}

Recent helioseismic probing of the solar interior has shown that the rotation rate of the Sun near the base of its convective zone changes with a period of roughly 1.3 years (Howe et al. 2000). The differential rotation of the Sun is one of the main ingredients of the dynamo located at the base of the convection zone, which generates the magnetic field that is observed at the solar surface. It is thus reasonable to ask whether the 1.3year periodicity also shows up in the surface manifestations of solar activity. There are two additional grounds to look for such a periodicity at the solar surface. One is that the periodicity has also been detected in variations of the interplanetary magnetic field and geomagnetic activity (Paularena et al. 1995; Szabo et al. 1995; Lockwood 2001) and in the solar wind speed (Richardson et al. 1994). The other is the fact that, based on an analysis of the same data, Antia \& Basu (2000) drew conclusions that contradict those of Howe et al. (2000). The presence or absence of this periodicity in solar surface data would strengthen or weaken the case for associated changes in the layers harbouring the dynamo.

We expect that any fluctuation in the dynamo process will manifest itself most clearly in relatively freshly emerged flux, i.e. in young active regions. Sunspots, due to their relatively

Send offprint requests to: N. A. Krivova,

e-mail: natalie@linmpi.mpg.de short lifetimes, are good tracers of young active regions. Here we inspect sunspot areas and sunspot numbers as representatives of the freshly emerged solar surface magnetic field for a periodicity around 1.3 years as well as its harmonics. Whereas the helioseismic data cover only four 1.3-year cycles between 1995 and 1999, the analysis by Lockwood (2001) extends over more than a century and suggests that the amplitude of the periodic signal may vary with time. The wavelet transform decomposes a time series into time-frequency space, thus enabling the determination of the frequency spectrum of the variations as a function of time. It is therefore ideally suited for our purpose.

Another periodicity in solar activity, namely that around 150-160 days, is also of interest in this connection. Variations with this period, first seen in the occurrence of high-energy solar flares (Rieger et al. 1984), have also been detected in sunspot areas (Lean \& Brueckner 1989; Lean 1990; Carbonell \& Ballester 1990, 1992; Oliver et al. 1998) and sunspot numbers (Lean \& Brueckner 1989). It is interesting to note that whether by coincidence or not, three times 150-160 days corresponds to $1.25-1.3$ years. Therefore, we also look at the possible relation between the 1.3-year and 156-day periodicities.

\section{Time series and the wavelet transform}

In this paper we consider two different records of solar activity. First, we analyze monthly averaged measurements of sunspot 
areas, $A_{\mathrm{s}}$. Such observations were regularly carried out by the Royal Greenwich Observatory starting in 1874, but were unfortunately stopped in 1976. As was shown by Fligge \& Solanki (1997), more recent measurements by different groups differ significantly from the earlier recordings and a correction factor must be introduced when pooling the data sets. For 1977-1999, we therefore use the recordings by Rome and Yunnan observatories scaled following Fligge \& Solanki (1997). The other data set we employ is that of Zürich relative sunspot numbers, $R_{\mathrm{z}}$. Monthly values of the sunspot number recorded between 1749 and 2001 are analyzed.

The wavelet transform is a powerful tool for analyzing localized variations of power within a time series and is finding ever-widening astronomical and geophysical applications (e.g., Torrence \& Compo 1998; Oliver et al. 1998; Fligge et al. 1999). Contrary to classical Fourier analysis that decomposes a signal into different sines and cosines which are not bounded in time, the wavelet transform uses wavelets characterized by scale (frequency) and position in time. It can be employed to analyze time series that contain non-stationary power at many different frequencies, i.e. it perfectly suits our purpose. Here we use the Morlet wavelet, consisting of a plane wave modulated by a Gaussian. This type of wavelet has turned out to be particularly appropriate for time series analyses. The choice of its width and frequency is dictated by the aim of a particular application: a narrow function (in time) gives good time resolution but poor frequency resolution, whereas broadening of the function results in a poorer localization in time, but allows a more accurate determination of the frequency. Further details can be found in the papers by Torrence \& Compo (1998) and Fligge et al. (1999).

\section{The 156-day periodicity}

By applying the wavelet transform to a time series sampled on a monthly basis one can study periods between two months and the total length of the time series. We first look for periods around 150-160 days.

A periodicity, peaked at about $154-158$ days, was first mentioned for high-energy solar flares (Rieger et al. 1984; Kiplinger et al. 1984; Bogart \& Bai 1985) and later revealed in the time series of sunspot areas (Lean \& Brueckner 1989; Lean 1990; Carbonell \& Ballester 1990, 1992) and numbers (Lean \& Brueckner 1989). To study this short-term variability in sunspot areas, Oliver et al. (1998) also took advantage of the wavelet transform. We can thus test our results against theirs, comparing the wavelet power spectra at the corresponding frequencies. The analysis by Oliver et al. (1998) has shown that the periodicity appears only near the maxima of some solar cycles which they interpreted as the manifestation of a periodic emergence of magnetic flux. The periodicity at 1.3 years has been detected in the rotation of the Sun near the base of its convection zone and thus should also be best visible as a periodic emergence of magnetic flux with this period. Hence a connection between the two periods is quite plausible.

The local wavelet power spectrum is plotted in Fig. 1. To facilitate comparison we have taken the frequency resolution of the wavelets to be consistent with that used by
Oliver et al. (1998). By and large, the results are quite similar to those of Oliver et al. (1998). In particular, the wavelet power spectra are almost identical for the period before 1980 . The periodicity during 1980-1985, however, is more pronounced in our plot and does not disappear after 1985, contrary to the conclusion reached by Oliver et al. (1998). Whether we interpolate over data gaps or not does not significantly affect the results. The discrepancy arises from the difference in the data sets employed for the period after 1976 when the Royal Greenwich Observatory stopped observations. As described in Sect. 2, we used a compilation from Rome and Yunnan data scaled following Fligge \& Solanki (1997). The uncorrected sunspot areas used by Oliver et al. (1998) are smaller than the corrected ones, which explains at least a part of the decrease in power after 1980 obtained by them. Other possible sources of this discrepancy are the inhomogeneity of the data sources since 1976 (we have only used Rome and Yunnan data, which are in good agreement with each other) and the fact that the data set employed by Oliver et al. (1998) ended in 1993. Note the shift in the power peak to longer periods around 1990.

\section{The 1.3-year periodicity and its relation to other periods}

In Fig. 2 we display the wavelet spectrum obtained with the same parameters as in Fig. 1 but for a much broader range of periods. Note a decrease in time resolution for longer periods. Along with the local maxima at $\sim 0.40-0.45$ year discussed in Sect. 3, the power spectrum has a maximum at about 1.21.3 years, whose amplitude varies strongly with time, however. The periodicity was particularly prominent during the interval 1920-1965 and then, although considerably weaker, appeared again in the 21 st and subsequent cycles (i.e. from roughly 1980 onwards). The wavelet transform using a wavelet with higher temporal resolution reveals that the power at this period is stronger around the phase of solar cycle maximum.

To determine the period corresponding to the maximum power with greater accuracy, we use Morlet wavelets with higher frequency (and hence lower temporal) resolution. The resulting local and global wavelet power spectra are plotted in Figs. $3 a$ and $b$, respectively. One can easily identify the maximum around 1.3 years. It is significant at a level of about $90 \%$, which is much higher than the 154-158 days period, and is the most significant peak shortward of 3.5 years. The precise position of the peak is 1.28 years, in good agreement with the period found from the helioseismic data $-0.78^{-1} \mathrm{yr}=1.28 \mathrm{yr}$ (Howe et al. 2000), which may be by chance, given the short duration of the helioseismic dataset. Interestingly, during the time interval over which the 1.3-year period is seen in the Sun's internal rotation rate power in the sunspot areas dataset is reduced, but remains significant at the $85 \%$ level. It is also worth noting that one third of $1.28 \mathrm{yr}$ makes 156 days and the maximum of power for the 154-158 day periodicity lies at 158 days.

A similar analysis of monthly records of sunspot numbers starting in the year 1749 , when monthly records first became available, also reveals the 1.3 -year period, at a confidence level of about $70 \%$, with only a weak dependence on the employed frequency resolution. We find maxima of global power 


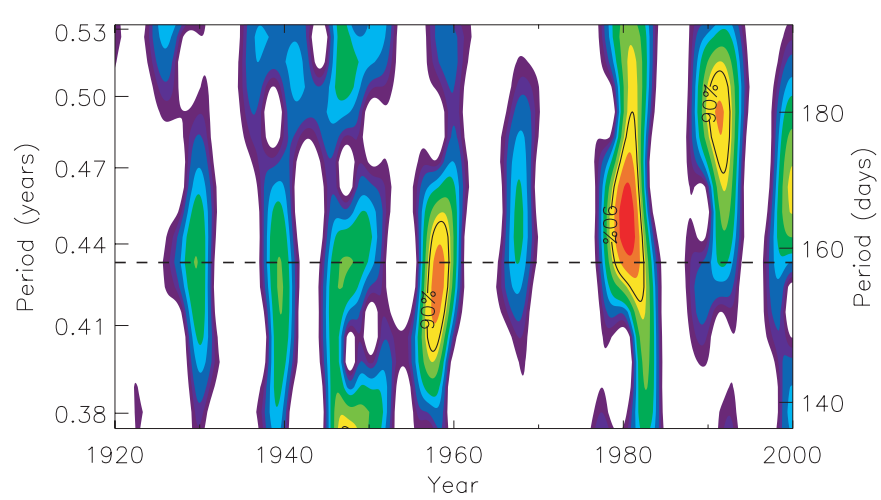

Fig. 1. The local wavelet power spectrum of the record of sunspot areas for periods between 138 and 194 days. White represents areas of little power, red those with the largest power, solid rings mark the $90 \%$ confidence level. The horizontal dashed line represents the Rieger period of 156 days and $1 / 3 \times 1.3$ years, which are almost identical.

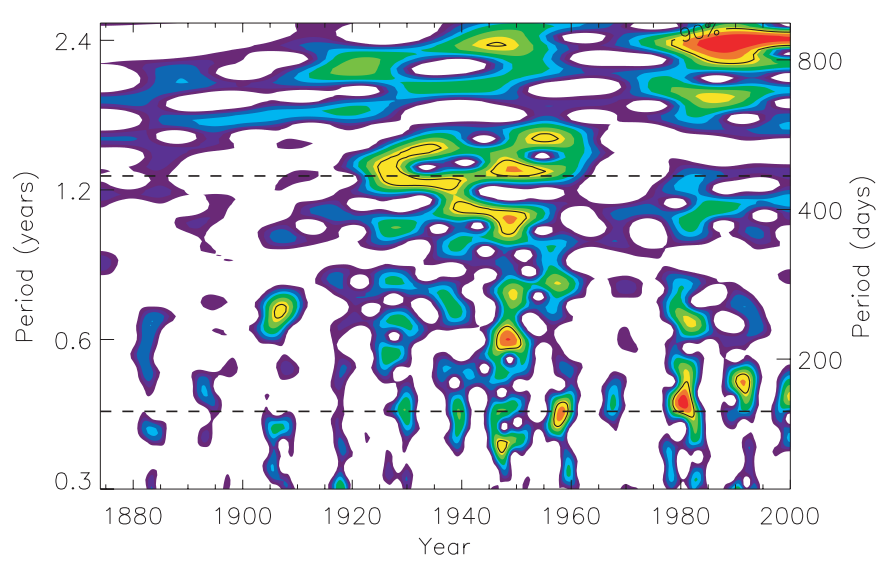

Fig. 2. The same as Fig. 1, but for a broader range of frequencies. The dashed lines are at 156 days and 1.28 years.

at $1.29 \mathrm{yr}$ and 157 days. Note that 1.29 years corresponds to exactly $3 \times 157$ days. Power in the 1.3 year peak is largest during the intervals 1750-1800,1830-1870 and after 1910, getting somewhat weaker again after 1960. There is also a tendency to shorter periods at earlier times, down to $\sim 1.13$ years in the 18 th century. We warn, however, that this portion of the data is the least reliable.

Other features that can be identified in Figs. 2 and 3 are a possible periodicity at $\sim 0.6-0.8$ years and a significant power at around 2.4-2.6 years. The 0.6-0.8-year modulation seems to include two periods: one at $0.65 \mathrm{yr}$, i.e. half of the 1.28-year period, and at $0.76 \mathrm{yr}(0.87 \mathrm{yr}$ for sunspot numbers), i.e. about twice the 156-day period. Note, however, that these periods, as well as the 156-day one, hardly stand out at high resolution (Fig. 3), possibly because the wavelets now do not have sufficient time resolution to distinguish between the activity minimum and maximum phases. The peak near 2.5 years, i.e. nearly twice the 1.3-year period, is distinguishable at a confidence level of about $80 \%$ and after 1980 is even more significant, at a level higher than $95 \%$. We refrain from discussing the peaks near 1 year since they may be influenced by seasonal effects.

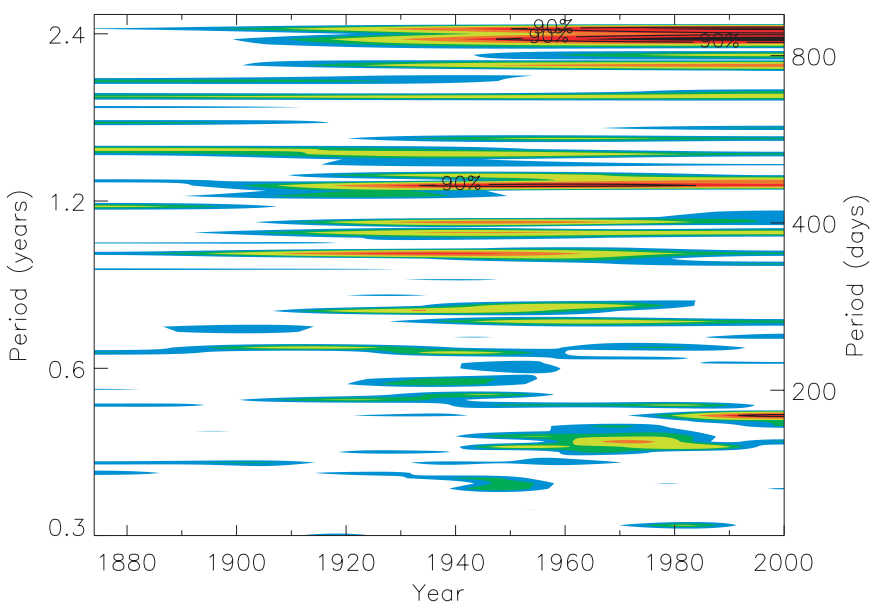

(a)

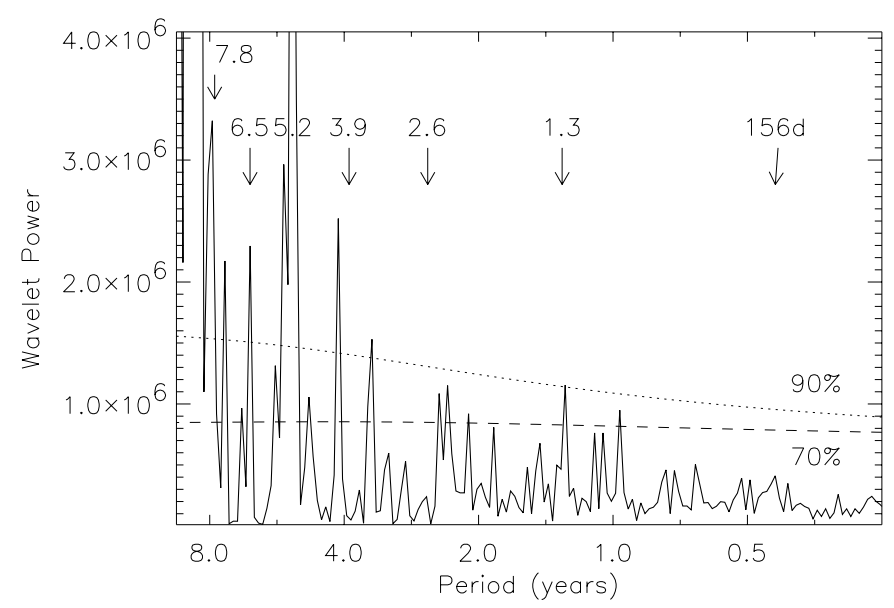

(b)

Fig. 3. a) The same as Fig. 2, but for a higher frequency resolution. b) Global wavelet power spectrum of sunspot areas plotted for a larger range of periods than shown in a). The dashed and dotted lines are the $70 \%$ and $90 \%$ confidence limits, respectively.

Figure 2 indicates that the power at the periods considered here is significantly larger after approximately 1920 and is strongest during cycle 18. At these times the sunspot areas were also large, suggesting the need to check for a connection between the two. For the sunspot numbers we also find that the periodicity is most prominent during times of higher solar activity, whereas it decays and sometimes disappears into the background during weak cycles. To check whether the correlation is real, we calculate 11-year running means of sunspot areas and of the wavelet power at the periods of interest.

In order to analyse the temporal variability of the power, high resolution in time is needed, which leads to a rather poor frequency resolution. If we then consider a narrow frequency interval around a given peak we find that the power is often greatly reduced, whereas a broad range contains also a lot of noise (see Fig. 2) and it sometimes becomes difficult to separate neighbouring periods. In Fig. 4 we therefore show the power deduced from sunspot areas for both cases as a function of time and compare it with the 11-year averaged sunspot area. In Fig. 4a we plot the power near 1.3 years over a 
narrow (0.04 years broad) frequency interval centred on 1.28 years (dot-dashed curve). The correlation with $A_{\mathrm{s}}$, although imperfect, is nevertheless clearly seen. For the broad frequency interval, considering just the 1.3 year interval (1.11.5 years) does not turn up a particularly good correlation with $A_{\mathrm{s}}$ or $R_{\mathrm{z}}$. We noticed, however, that the amplitude of the 2.6 year peak shows in some ways a complementary behaviour in that it is strong at times when the 1.3-year peak is weak and vice versa. In Fig. $4 \mathrm{~b}$ we therefore plot the sum of the power in both peaks $(1.3+2.6$ years; dot-dashed curve $)$. Figure 5 displays the 11-year smoothed sunspot number record (solid) together with the power in the narrow 1.3 year band (Fig. 5a) and the combined power in the broad $1.3+2.6$ year bands (Fig. $5 b$ ) computed from sunspot numbers (dot-dashed curves).

The maximum value of the cross-correlation between 1.3 year power and sunspot area/number is approximately $0.75-0.8$, reached at a time lag of approximately one cycle. Hence, a variation in the power at the 1.3 -year period precedes the change in the sunspot areas or numbers. The maximum in the cross correlation coefficient as a function of time lag is, however, rather broad, with the coefficient at a zero lag being lower by only $0.05-0.1$. Thus we do not attach particular significance to the presence of a time lag.

It is worth mentioning that a periodicity of around 1.4 years was found by Silverman \& Shapiro (1983) in a data set of Swedish visual aurorae for the period 1721 to 1943 . The power at this period was found to be modulated over 65 years, with strong maxima at 1754-1774 and 1822-1843 and a weaker broad maximum during 1867-1900, which agrees reasonably with maxima in the dot-dashed curve in Fig. 5b. The agreement with Fig. 5a may be less good since some peaks may be missed when considering a narrow interval, as the positions (periods) of the peaks vary with time.

We repeated the correlation analysis with respect to the 156-day period and obtained very similar results. The power in this peak (averaged over a period interval of 7 days) is represented by the dashed curves in Figs. $4 \mathrm{a}$ and $5 \mathrm{a}$. The combined power in the 156-day band and in the third harmonic of the 2.6-year period, the 312-day band, is plotted in Figs. 4b and $5 \mathrm{~b}$. Furthermore, we calculated the correlation coefficient between power at the longer period $(1.3$ or $1.3+2.6)$ and the shorter one $(156$ or $156+312)$, which turned out to be about $0.7-0.8$. Similar correlations are obtained between the 156-day period power and the sunspot area and number records. The probability that the correlations are due to chance is less than $5 \times 10^{-5}$ for sunspot number and $5 \times 10^{-3}$ for sunspot area (the larger probability is because it is a shorter time series). Note that the overall shape of the curves in Figs. 4 and 5 is robust as far as perturbations of the period intervals are concerned (in Figs. $4 \mathrm{~b}$ and $5 \mathrm{~b}$ we took $0.2,0.3,0.4$ and $0.8 \mathrm{yr}$ broad intervals at 156 days, 312 days, $1.28 \mathrm{yr}$ and $2.6 \mathrm{yr}$, respectively). The correlation coefficients also do not depend strongly on these choices.

Since a part of the "noise" in the sunspot data is solar in origin (fluctuations in the number and area of sunspots from day to day) it may also scale with $A_{\mathrm{s}}$ and $R_{\mathrm{z}}$. In this case the high significance of the correlations between, e.g., 1.3 years and 154-158 days would have to be questioned. We find,
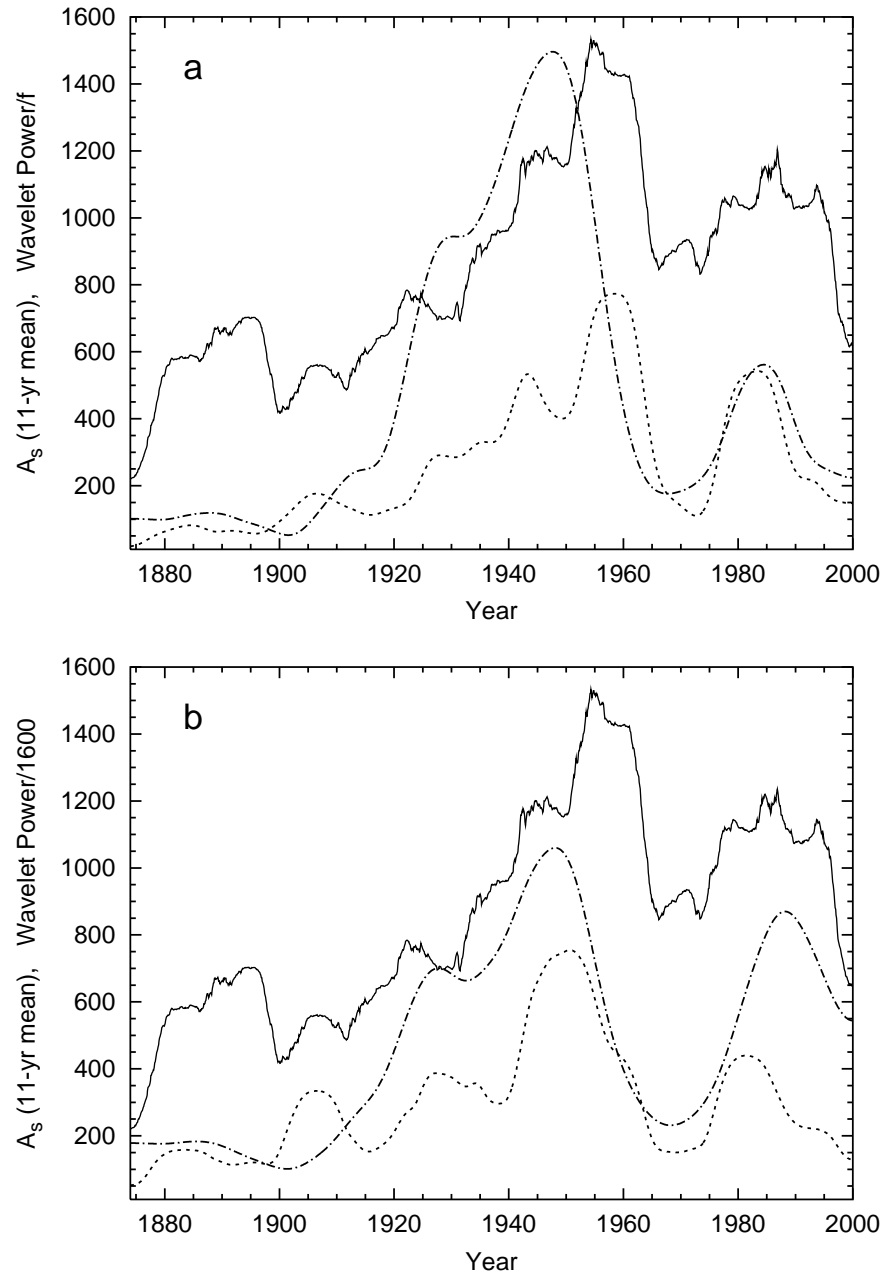

Fig. 4. The 11-year running mean of sunspot areas (solid line) and of the corresponding wavelet power spectrum at the periods of $1.3 \mathrm{yr}$ (dot-dashed line) and 156 day (dotted line). The wavelet power has been divided by a factor of $f$ to allow it to be plotted on the same scale. In Fig. 4a, dotted and dot-dashed curves have been normalized by different values $f$ ( $f=1000$ and 2000, respectively), in Fig. 4b $f=1600$. a) Narrow intervals (which are 0.04 and 0.02 yr broad, respectively) centered at the frequencies of maximum power. b) The same, but for broad intervals and the combined power in the peaks at $1.28+2.45 \mathrm{yr}$ and $156+312$ days, respectively $(0.4+0.8 \mathrm{yr}$ and $0.2+0.3$ yr broad).

however, that the noise (which we take to be the power at frequencies between the peaks) correlates only at a level of $0.2-0.4$ with $A_{\mathrm{s}}$ and $R_{\mathrm{z}}$, with the exact value of the correlation coefficient (and the phase of the "noise" power) being strongly dependent on the exact frequency interval chosen. We therefore conclude that the correlation between the power at the 1.3 years and 156 days is not an artifact of a dependence of the noise on sunspot number or area.

A striking feature of the power spectrum shown in Fig. 3 is the presence, apart from a few other maxima, of all multiples of the 1.3-year peak. Besides the peak at close to 2.6 years mentioned above, the higher multiples at 3.9, 5.2, 6.5, 7.8 and 9.1 years are seen at confidence levels higher than $90 \%(75 \%$ for sunspot numbers, see Table 1). Note, however, that the "2.6year peak" in the global spectrum lies closer to 2.4 years, while the "3.9-year peak" is actually found at 4.1 years. This does not 

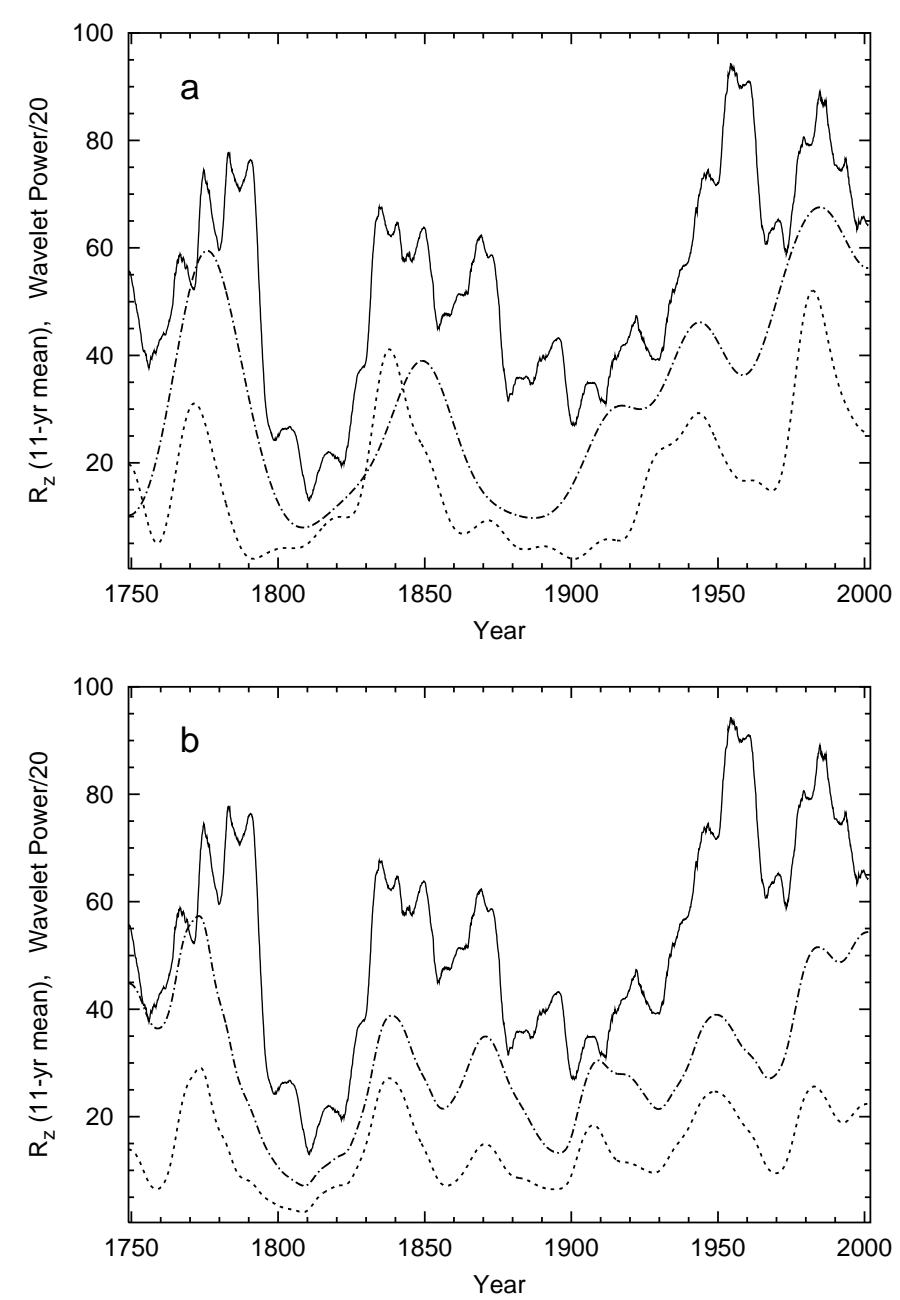

Fig. 5. The same as Fig. 4, but for sunspot number. The wavelet power has been divided by 20 to allow for a better comparison.

rule out that these periods are related to each other. For all these periods, just as for the solar cycle itself, both the amplitude and frequency of the peaks change with time. Since the amplitudes of the various peaks do not vary completely in phase (see Figs. 2 and 3), the averaged frequencies do not need to be exact multiples of a basic frequency. Hence it is still possible that these peaks are related to the 1.3-year period.

Could the peaks at 5.2-1.3 years be harmonics of the sunspot cycle? For the often quoted length of 11.2 years this would in general not be the case (e.g., 11.2/1.28 = 8.75). However, the amplitudes and periods of all the peaks vary with time. This means that they should not be compared with the simple time average of the sunspot cycle period, but rather the average amplitude of the weighted period of the cycle, since it is this quantity which is returned by the wavelet analysis. We find that the resulting weighted period depends only slightly on whether the sunspot number or the amplitude of the 1.3-year peak is used as a weight, which is not unexpected judging from Figs. 4 and 5. If we weight the sunspot cycle period obtained by applying wavelets (e.g., Fligge et al. 1999) with the power in the 1.3 year peak we obtain, averaged over time, 10.28 years for the sunspot areas data set.
Table 1. Periods, $P$, and significance, $s$, of the possible $n$th subharmonic of the 1.3-year period revealed by the wavelet analysis of sunspot areas $A_{\mathrm{s}}$ and numbers $R_{\mathrm{z}}$.

\begin{tabular}{cccrcr}
\hline \hline & & \multicolumn{2}{c}{$A_{\mathrm{s}}$} & \multicolumn{2}{c}{$R_{\mathrm{z}}$} \\
$n$ & $n \times 1.3$ & $P$ & $s(\%)$ & $P$ & $s(\%)$ \\
\hline 2 & 2.6 & $2.35 / 2.45$ & 85 & $2.35 / 2.76$ & 65 \\
3 & 3.9 & 4.12 & $>99$ & 4.16 & 80 \\
4 & 5.2 & 5.23 & $>99$ & 5.40 & $>99$ \\
5 & 6.5 & 6.49 & 95 & 6.42 & 75 \\
6 & 7.8 & 7.89 & 95 & 7.97 & 90 \\
7 & 9.1 & 8.99 & $>99$ & & \\
8 & 10.4 & 10.46 & $>99$ & 10.79 & $>99$ \\
\hline
\end{tabular}

As can be seen from Table 2 this means that the deduced 1.28 year period could in principle be the 8 th harmonic of the solar cycle. For the sunspot number data this identification is not so clear (Table 3) and does not improve if we restrict our analysis to more recent and reliable data. We repeat this analysis for the other peaks that we tentatively identify as multiples of 1.3 years, weighting the cycle length with the current amplitude of the relevant peak. The results are listed in Tables 2 and 3. Only the peak around 5.2-5.4 years qualifies as a harmonic of the solar cycle. Hence, most of the peaks between 2.4 and 9.1 years tentatively identified as subharmonics of 1.3 years cannot be harmonics of the sunspot cycle, which supports the idea that the 1.3-year period is independent of the activity cycle. Given the good correlation with the sunspot cycle amplitude (Figs. 4 and 5) we cannot completely rule out, however, that the 1.3-year period itself (and hence probably also the 156-day period) is a harmonic of the 11-year cycle.

\section{Conclusions}

Wavelet analysis of sunspot areas and sunspot numbers shows that the periodicity of 1.3 years revealed in the rotation rate of the Sun at the base of the convection zone is also present in these records of solar activity. We also find significant power at all multiples of 1.3 years up to 10.4 years, which is nearly the period of the solar cycle. This suggests that variations in the rotation rate do indeed have an influence on the workings of the solar dynamo. We cannot rule out, however, that the 1.3 year period seen in sunspot data is simply the 8th harmonic of the sunspot cycle.

We also point out that the 154-158-day Rieger period exhibited by solar flares could be the third harmonic of the 1.3 -year period (which is 1.28 years in the sunspot data, and whose third harmonic lies at 156 days). We also confirm the presence of the 154-158-day period in variations of sunspot areas and numbers, although with lower significance than the 1.3-year period, when averaged over the whole sunspot areas time series. In contrast to Oliver et al. (1998) we find that the 156-day period persists into the most recent cycles.

The wavelet analysis clearly reveals that the power at the 1.3-year and the 156-day periods fluctuates considerably with time, being stronger during stronger sunspot cycles. The high correlation between all three quantities allows three interpretations. 1. The 1.3-year and 156-day periods are harmonics of 
Table 2. Periods, $P$, of possible subharmonics of the 1.3 year period, weighted solar cycle length, $l_{\mathrm{w}}$, and their ratio. Sunspot areas data.

\begin{tabular}{ccc}
\hline \hline$P$ & $l_{\mathrm{w}}$ & $l_{\mathrm{w}} / P$ \\
\hline 1.28 & 10.28 & 8.03 \\
$2.35 / 2.45$ & 10.27 & $4.37 / 4.19$ \\
4.12 & 10.55 & 2.56 \\
5.23 & 10.33 & 1.98 \\
6.49 & 10.37 & 1.60 \\
\hline
\end{tabular}

Table 3. The same as Table 2, but for sunspot number.

\begin{tabular}{ccc}
\hline \hline $\mathrm{P}$ & $l_{\mathrm{w}}$ & $l_{\mathrm{w}} / P$ \\
\hline $1.13 / 1.29$ & 10.57 & $9.35 / 8.19$ \\
$2.35 / 2.76$ & 10.75 & $4.57 / 3.89$ \\
4.16 & 10.92 & 2.63 \\
5.40 & 10.88 & 2.01 \\
\hline
\end{tabular}

the sunspot cycle. 2. The dynamo is affected by the 1.3-year period of rotation variations and finally it is this effect which is responsible for the 156-day period of flux emergence and flare activity. 3 . It is the dynamo which excites the 1.3 year periodicity in solar rotation, so that this and the other periodicities seen in the sunspot data all have a common source.

A periodicity at 1.3 years has also been detected in variations of geomagnetic activity and interplanetary magnetic field (Paularena et al. 1995; Szabo et al. 1995; Lockwood 2001). Is it related to the 1.3 year period in the sunspot numbers and the internal rotation? According to the recent analysis of Lockwood (2001), the period was found to be quite prominent for the interval 1995-2000, whereas almost no peak at this frequency was seen in the power spectrum for 1943-1959 (his Fig. 16). Our analysis of sunspot data reveals that the periodicity is much stronger for the latter time interval. This indicates that the periodicity detected by Lockwood has other or at least additional sources. This is not entirely surprising, since according to Sheeley (1992) and Solanki et al. (2000) the open magnetic flux which underlies the interplanetary field lives for multiple years at the solar surface, so that a 1.3 -year periodicity would tend to be smoothed away.

Acknowledgements. We are grateful to M. Fligge for putting the data sets at our disposal. Wavelet software was provided by C. Torrence and G. Compo, and is available at URL: http://paos.colorado.edu/research/wavelets/

\section{References}

Antia, H. M., \& Basu, S. 2000, ApJ, 541, 442

Bogart, R. S., \& Bai, T. 1985, ApJ, 299, L51

Carbonell, M., \& Ballester, J. L. 1990, A\&A, 238, 377

Carbonell, M., \& Ballester, J. L. 1992, A\&A, 255, 350

Fligge, M., \& Solanki, S. K. 1997, Sol. Phys., 173, 427

Fligge, M., Solanki, S. K., \& Beer, J. 1999, A\&A, 346, 313

Howe, R., Christensen-Dalsgaard, J., Hill, F., et al. 2000, Science, 287, 2456

Kiplinger, A. L., Dennis, B. R., \& Orwig, L. E. 1984, BAAS, 16, 891

Lean, J. 1990, ApJ, 363, 718

Lean, J. L., \& Brueckner, G. E. 1989, ApJ, 337, 568

Lockwood, M. 2001, J. Geophys. Res., 106, 16021

Oliver, R., Ballester, J. L., \& Baudin, F. 1998, Nature, 394, 552

Paularena, K. I., Szabo, A., \& Richardson, J. D. 1995, Geophys. Res. Lett., 22, 3001

Richardson, J. D., Paularena, K. I., Belcher, J. W., \& Lazarus, A. J. 1994, Geophys. Res. Lett., 21, 1559

Rieger, E., Kanbach, G., Reppin, C., et al. 1984, Nature, 312, 623

Sheeley, N. R. 1992, in The Solar Cycle, ed. K. L. Harvey (San Francisco), ASP Conf. Ser., 27, 1,

Silverman, S. M., \& Shapiro, R. 1983, J. Geophys. Res., 88, 6310

Solanki, S. K., Schüssler, M., \& Fligge, M. 2000, Nature, 408, 445

Szabo, A., Lepping, R. P., \& King, J. H. 1995, Geophys. Res. Lett., 22,1845

Torrence, C., \& Compo, G. P. 1998, Bull. Am. Meteorol. Soc., 79, 61 